\title{
White Matter Injury after Cerebral Ischemia in Ovine Fetuses
}

\author{
KATHERINE H. PETERSSON, HALIT PINAR, EDWARD G. STOPA, RONALD A. FARIS, \\ GRAZYNA B. SADOWSKA, R. CHOUDARY HANUMARA, AND BARBARA S. STONESTREET \\ Department of Pediatrics [K.H.P., G.B.S., B.S.S.] and Pediatric Pathology [H.P.], Department of \\ Pathology (Division of Neuropathology) [E.G.S.] and Pediatrics [R.A.F.], Brown University School of \\ Medicine, Providence, Rhode Island 02906; Women and Infants' Hospital of Rhode Island and Rhode \\ Island Hospital, Providence, Rhode Island 02905; Department of Computer Science and Statistics, The \\ University of Rhode Island, Kingston, Rhode Island 02881 [R.C.H.], U.S.A.
}

\begin{abstract}
The effects of cerebral ischemia on white matter changes in ovine fetuses were examined after exposure to bilateral carotid artery occlusion. Fetal sheep were exposed to $30 \mathrm{~min}$ of ischemia followed by 48 (I/R-48, $n=8)$ or $72(\mathrm{I} / \mathrm{R}-72, n=10) \mathrm{h}$ of reperfusion or control sham treatment (control, $n=4)$. Serial coronal sections stained with Luxol fast blue/hematoxylin and eosin were scored for white matter, cerebral cortical, and hippocampal lesions. All areas received graded pathologic scores of 0 to 5 , reflecting the degree of injury where $0=0 \%, 1=1 \%$ to $25 \%, 2=26 \%$ to $50 \%, 3=51 \%$ to $75 \%, 4=76 \%$ to $95 \%$, and $5=96 \%$ to $100 \%$ of the area damaged. Dual-label immunofluorescence using antibodies against glial fibrillary acidic protein (GFAP) and myelin basic protein (MBP) were used to characterize white matter lesions. Basic fibroblast growth factor (FGF-2) was measured in the frontal cortex by ELISA. Results of the pathologic scores showed that the white matter of the I/R-72 $(2.74 \pm 0.53$, mean \pm SEM) was more $(p<0.05)$ damaged when compared with the control $(0.80 \pm 0.33)$ group. Cortical lesions were greater $(p<0.05)$ in the I/R-48 $(2.12 \pm 0.35)$ than the control $(0.93 \pm 0.09)$ group. White matter lesions were characterized by reactive GFAP-positive astrocytes and a loss of
\end{abstract}

\section{ABSTRACT}

MBP in oligodendrocytes. The ratio of MBP to GFAP decreased $(p<0.05)$ as a function of ischemia, indicative of a proportionally greater loss of MBP than GFAP. FGF-2 concentrations were higher $(p<0.05)$ in the I/R-72 than the control group and there was a direct correlation between the pathologic scores (PS) and FGF-2 concentrations (FGF-2 $=\mathrm{e}^{(1.6 \text { PS-0.90) }}+743, n=17, r=$ $0.73, p<0.001)$. We conclude that carotid artery occlusion results in quantifiable white matter lesions that are associated with a loss of MBP from myelin, and that FGF-2, a purported mediator of recovery from brain injury in adult subjects, increases in concentration in proportion to the severity of brain damage in the fetus. (Pediatr Res 51: 768-776, 2002)
Abbreviations
FGF-2, basic fibroblastic growth factor
EcoG, electrocorticogram
I/R-48, ischemia and $48 \mathrm{~h}$ of reperfusion
I/R-72, ischemia and $72 \mathrm{~h}$ of reperfusion
GFAP, glial fibrillary acidic protein
MBP, myelin basic protein

Hypoxic/ischemic brain injury is the single most important neurologic problem occurring in the perinatal period. There is substantial evidence to suggest that a major component of brain injury is related to ischemia alone or hypoxia/ischemia (1-3). Although many studies have characterized the neuronal outcome to a variety of ischemic insults, white matter injury has not been well studied (4).

Received October 12, 2001; accepted January 23, 2002

Correspondence and reprint requests: Barbara S. Stonestreet, M.D., Brown University School of Medicine, Department of Pediatrics, Women and Infants' Hospital of Rhode Island, 101 Dudley Street, Providence, RI 02905, U.S.A.; e-mail: bstonest@wihri.org

Supported by the National Institute of Child Health and Human Development Grant R01-HD-34618.

DOI: 10.1023/01.PDR.0000017486.64723.D1
Nonetheless, white matter injury has been described recently in neonatal (5-7) and adult rats (8) using various forms of carotid artery occlusion. Several studies have found white matter injury in fetal sheep that were subjected to hypoxemia (9), systemic hypotension (10), bilateral carotid artery occlusion $(11,12)$, and repeated umbilical cord occlusion (13-16). However, these studies have not quantified the white matter lesions or defined the pattern of white matter injury in multiple brain regions. Although neuronal injury arising from bilateral carotid artery occlusion in the ovine fetus has been extensively described (17-19), the accompanying white matter lesions have not been thoroughly investigated or quantified. Therefore, in this study, to further elucidate the pathology of cerebral cortical and white matter lesions arising from cerebral ischemia in the late gestation ovine fetus, we have investigated markers 
of both neurons and glia (astrocytes and oligodendrocytes) to identify specific cellular damage. Pathologic evaluation was performed using stains that allowed for optimal evaluation and quantification of both the white matter and the cerebral cortex. Using double-label immunofluorescence, white matter lesions were characterized by evaluating the presence and appearance of GFAP and MBP. A glial intermediate cytoskeletal protein rich in aspartic and glutamic acid, GFAP is located abundantly in white matter and subpial astrocytes (20). MBP is a major structural protein in myelin that recently has been postulated to play a role in myelinogenesis (21).

FGF-2 is known to be part of an endogenous repair system that can rescue or support injured brain tissue. Increased expression of FGF-2 has been demonstrated after cerebral ischemia in adult subjects (22-25). In vivo, exogenously administered FGF-2 has been shown to attenuate ischemic brain injury in the neonate $(26,27)$ and the adult $(27-31)$. However, the effects of in utero cerebral ischemia on changes in FGF-2 concentration have not been examined in the cerebral cortex of the fetus.

Given the above considerations, we quantified the pathologic effects of in utero cerebral ischemia on white matter lesions in the ovine fetus. To study this, we examined the effects of in utero ischemic brain injury on cerebral cortical and white matter lesions in six coronal sections from the rostral to the caudal aspect of the brain of ovine fetuses at $126 \mathrm{~d}$ of gestation. We used the method of Gluckman and colleagues (18) to induce cerebral ischemia by bilateral occlusion of the carotid arteries after ligation of the vertebral-occipital anastomoses. This has proven to be a very reproducible model of in utero brain ischemia $(18,19,32)$. The severity of the damage in both the white matter and cerebral cortex was examined using a modification of the established scoring system by Gluckman and colleagues (19) on whole brain sections stained with Luxol fast blue/hematoxylin and eosin. White matter lesions arising from the ischemic insult were further characterized by staining for myelin. Finally, FGF-2 concentration was measured and correlated with cerebral cortical injury.

\section{MATERIALS AND METHODS}

This study was approved by the Institutional Animal Care and Use Committees of Brown University and Women and Infants' Hospital of Rhode Island and in accordance with the National Institutes of Health guidelines for use of experimental animals.

Animal preparation. Surgery was performed on 22 mixedbreed ewes from 118 to $121 \mathrm{~d}$ of gestation. Anesthesia was induced in the ewes by an intramuscular injection of ketamine and maintained with 1-2\% halothane and oxygen after endotracheal intubation. The uterus was exposed by a midline abdominal incision, and the fetus operated on through a hysterotomy with minimal loss of amniotic fluid $(33,34)$. Polyvinyl catheters were placed into a brachial artery and advanced to the thoracic aorta for blood sample withdrawal, into a femoral artery and advanced into the thoracic aorta for heart rate and blood pressure monitoring, and into a brachial vein for drug administration. An amniotic fluid catheter was placed for pressure monitoring to correct fetal arterial pressures.

The fetal carotid arteries were exposed caudal to the angle of the mandible (19). The vertebral-occipital anastomoses proximal to occluder placement were ligated to restrict blood flow from the vertebral circulation $(17,19)$. The lingual arteries were ligated to restrict noncerebral blood flow. Two inflatable 4-mm vascular occluders (In Vivo Metric, Healdsburg, CA, U.S.A.) were placed around each carotid artery $(17,19)$. For EcoG measurement, two pairs of stainless steel screws (size 0-80, Small Parts, Inc., Miami Lakes, FL, U.S.A.) were implanted via burr holes into the fetal skull and onto the dura overlying the parietal cortex $3 \mathrm{~mm}$ posterior to the bregma and $3 \mathrm{~mm}$ anterior to the intersection of the sagittal and occipital sutures, with each placed $15 \mathrm{~mm}$ lateral to the sagittal suture with a reference electrode sewn to the scalp as previously described $(18,19,35)$. The stainless steel screws were connected to the recorder by shielded polyvinyl chloride-insulated wires $0.25 \mathrm{~mm}$ in diameter (Alpha Wire Co., Elizabeth, NJ, U.S.A.). The EcoG was performed in the fetal sheep to determine that the EcoG became isoelectric during the cerebral ischemia (19).

The ewes were permitted a recovery of 5-8 d after surgery. The vascular catheters were flushed every other day with heparinized saline $(10 \mathrm{U} / \mathrm{mL})$ to maintain patency. Antibiotics (1 $\mathrm{g}$ ampicillin and $10 \mathrm{mg}$ amikacin) were administered to the ewe intramuscularly daily for $3 \mathrm{~d}$ after surgery. In addition, the fetus was given ampicillin $(100 \mathrm{mg} / \mathrm{kg})$ and amikacin $(5 \mathrm{mg} /$ $\mathrm{kg}$ ) on each day the catheters were flushed. Studies were performed on the fetus if the fetal arterial blood gases were within the normal range $\left(\mathrm{pH}>7.30, \mathrm{PaO}_{2}>18\right.$, and $\mathrm{PaCO}_{2}<60$ $\mathrm{mm} \mathrm{Hg}$ at $39.5^{\circ} \mathrm{C}$ ). None of the ewes exhibited signs of infection between the initial instrumentation and the experimental studies. The temperatures of the sham-operated ewes were the same as those of the experimental groups $\left(38.4-38.7^{\circ} \mathrm{C}\right)$.

Experimental protocol and methodology. Five to eight days after surgery, animals were randomly assigned to one of three groups: $30 \mathrm{~min}$ of ischemia followed by $48 \mathrm{~h}$ reperfusion $(\mathrm{I} / \mathrm{R}-48, n=8), 30 \mathrm{~min}$ of ischemia followed by $72 \mathrm{~h}$ of reperfusion (I/R-72, $n=10$ ), or control sham treatment (control, $n=4)$. Cerebral ischemia was induced by inflating the carotid artery occluders with $0.9 \% \mathrm{NaCl}$ for $30 \mathrm{~min}$. In the control sham group, the occluders were not inflated. An isoelectric EcoG was used to ensure the reproducibility of cerebral ischemia (19). Arterial $\mathrm{pH}$, blood gases, heart rate, mean arterial blood and amniotic fluid pressures, and plasma glucose and lactate concentrations were measured on the fetus at baseline, $15 \mathrm{~min}, 1,2,24$, and $48 \mathrm{~h}$ and/or $72 \mathrm{~h}$ after cerebral ischemia or control sham treatment.

Heart rate and mean arterial blood and amniotic fluid pressures in fetal sheep were measured with pressure transducers (model 1280 C, Hewlett-Packard, Lexington, MA, U.S.A.) and recorded on a polygraph (model 17758 B Series, Hewlett Packard). For fetal EcoG measurements, the parietal EcoG was amplified, filtered, and recorded by a Grass Instruments recording system (30-Hz low-pass filter, 7 PI preamplifier, DC driver, model 7D, Grass Instruments Co., Quincy, MA, U.S.A.). The 
EcoG was monitored and intermittently recorded. Blood gases and $\mathrm{pH}$ were measured on a Corning blood gas analyzer (model 238, Corning Scientific, Medford, MA, U.S.A.) at $39.5^{\circ} \mathrm{C}$ for the fetus and $38.5^{\circ} \mathrm{C}$ for the ewe. Plasma glucose and lactate concentrations were determined in duplicate using a glucose/ lactate analyzer (YSI 2300 STAT, YSI Inc., Yellow Springs, OH, U.S.A.).

Brain removal and pathologic methods. At the end of the reperfusion period, the ewe was given i.v. pentobarbital (15-20 $\mathrm{mg} / \mathrm{kg}$ ) to achieve a surgical plane of anesthesia. A hysterotomy was performed and the fetus was withdrawn from the uterus with the umbilical circulation intact. The brain was rapidly removed and a portion of one frontal cortex obtained, immediately frozen in liquid nitrogen, and stored at $-80^{\circ} \mathrm{C}$ until analysis for FGF-2. Samples were alternatively taken from the right or left frontal cerebral cortex within each study group. The remainder of the brain was placed in $10 \%$ formalin. The ewe was then killed with pentobarbital (100-200 mg/kg). Formalin-fixed whole brains were coronally cut at 1 -cm intervals into six serial brain slices and paraffin embedded. The brain regions located within each slice are as follows: slice 1 (S1) was prefrontal cortex, slice 2 (S2) was frontal cortex, slice 3 (S3) was posterior medial eminence, slice 4 (S4) was parietal-occipital junction, slice 5 (S5) was occipital pole, and slice 6 (S6) was cerebellum and brainstem. Eight-micrometer-thick histologic sections from each paraffin-embedded block were routinely deparaffinized, rehydrated, and stained with Luxol fast blue in combination with hematoxylin and eosin to clearly delineate white matter lesions. The well-established scoring system of Gluckman and colleagues (19) was modified to include both white matter and neuronal scores. The wholemount coronal sections were scored by two pathologists, who were not aware of the treatment groups, for white matter, cerebral cortical, and hippocampal lesions. Myelin degradation and reactive gliosis were indicative of white matter damage $(19,36)$. Nuclear pyknosis, cytoplasmic reddening and condensation, and hyperchromatism were indicative of neuronal injury. The pathologists scored the most severely injured area of each section using the following pathologic scoring system $(0-5)$, where $0=0 \%, 1=1 \%$ to $25 \%, 2=26 \%$ to $50 \%, 3=$ $51 \%$ to $75 \%, 4=76 \%$ to $95 \%$, and $5=96 \%$ to $100 \%$ of the area damaged.

Double-label immunofluorescence. Dual-label immunofluorescence using antibodies against GFAP and MBP were used to further characterize white matter lesions. The coronal sections analyzed were those that contained the hippocampus. Formalin-fixed, paraffin-embedded sections were routinely deparaffinized and rehydrated. Double-label immunofluorescence analysis was performed according to published procedures (37) using affinity-purified, subclass-specific secondary antibodies (Pierce Chemical, Rockford, IL, U.S.A.). To visualize reactive astrocytes, sections were incubated with rabbit anti-cow GFAP primary antibody and reacted with donkey anti-rabbit IgG conjugated to FITC (Pierce Chemical). The sections were then incubated with mouse anti-MBP primary antibody (Biogenex, San Ramon, CA, U.S.A.), reacted with biotinylated goat anti-mouse IgG ( $\mathrm{Fc}$ fragment specific) secondary antibody (Pierce Chemical) and detected using strepta-
vidin/Texas red (Vector Laboratories, Burlingame, CA, U.S.A.). Sections were examined with a Nikon Microphot FX microscope (Nikon, Tokyo, Japan) equipped with an epifluorescence condenser and a 35-mm camera. Sections were photographed with Kodak Tmax 100 film (El 200, Eastman Kodak, Rochester, NY, U.S.A.). Photomicrographs were taken of representative areas, with or without lesions, in the deep white matter bordering the periventricular space in each group. GFAP and MBP colors were extracted from scanned fluorescent micrographs in Adobe Photoshop (Adobe Systems, Mountain View, CA, U.S.A.). Resulting images were converted to gray-scale images and analyzed using NIH Image (version 1.51, National Institutes of Health) to determine the percentage of MBP and GFAP within each lesion. The ratio of MBP to GFAP was then calculated. An equivalent neuroanatomically matched area was selected for each region from the control sham group.

FGF-2 assay. The protein concentration of FGF-2 was measured in frontal cortex by ELISA analysis. Frozen samples were available from 7 of 8 fetuses in the I/R- 48 group, 6 of 10 fetuses in the I/R-72 group, and 4 of 4 fetuses in the control group. Frozen samples of frontal cortex were homogenized on ice in buffer consisting of $20 \mathrm{mM}$ Tris-HCL, $\mathrm{pH} 7.4 ; 2.0 \mathrm{M}$ $\mathrm{NaCl} ; 1 \mathrm{mM}$ EDTA; $1 \mathrm{mM}$ EGTA; $0.5 \%$ deoxycholate; $1 \%$ Igepal; proteinase inhibitor cocktail ( $1 \mathrm{mM}$ phenylmethyl sulfonyl fluoride and $1 \mu \mathrm{g} / \mathrm{mL}$ of each of the following: aprotinin, leupeptin, and pepstatin A). The samples were centrifuged at $14,000 \mathrm{rpm}$ for $30 \mathrm{~min}$ at $4^{\circ} \mathrm{C}$ and the total protein content of clarified supernatants was determined with a commercially available assay kit (Pierce Chemical). The FGF-2 content of the supernatants was determined by ELISA using a human FGF-2 immunoassay (Quantikine, R \& D Systems, Minneapolis, MN, U.S.A.). The assay procedures were all performed in accordance with the manufacturer's specifications. An ELISA scanner (Titertek Multiscan Plus MKII, ICN, Costa Mesa, CA, U.S.A.) was used to measure the OD of total protein at $562 \mathrm{~nm}$ and the OD of FGF-2 at $450 \mathrm{~nm}$.

Statistical analysis. All results were expressed as mean \pm SEM. Serial measurements of physiologic variables were compared by two-factor ANOVA for repeated measures with group and time as the factors (38). If a significant difference was found by ANOVA, the variables were further compared by the Bonferroni two-group $t$ test. To further describe and enhance the statistically significant treatment by time and/or timegroup interactions, separate ANOVA for repeated measures were performed within each group. If a significant difference was found by ANOVA, the Newman-Keuls post hoc test was used to identify specific differences within the groups.

The brain scores were analyzed by ANOVA for repeated measures for three factors, with slice, region, and group as the factors. A repeated-measures ANOVA was applied to the brain slices, because multiple slices were examined within the same brain. Initially, the pathologists were analyzed as an additional factor in the model. This analysis demonstrated that there were no statistically significant differences between the two pathologists. Therefore, the scores by both pathologists were averaged to obtain the final scores for each brain section. Although the scores represent ordinal rather than continuous data, we 
used parametric ANOVA procedures to analyze the scores rather than nonparametric statistical analysis. Because the averages of the pathologists' scores were used, which approached normality and examination of residuals indicated that the normality assumption was appropriate. Nonparametric procedures have limited power when the sample sizes are small, and our data required a repeated-measures, mixed-model analysis, for which established nonparametric procedures were not available.

One-way ANOVA was used to compare the ratio of MBP to GFAP, and FGF-2 concentrations among the groups. If a significant difference was found by one-way ANOVA, the Newman-Keuls post hoc test was used to identify specific differences among the groups. Correlational analysis also was used to compare the frontal cortex FGF-2 concentration to the pathologic score in the same animals. The calculations were done using the Statistical Analysis Program (SAS Institute, Cary, NC, U.S.A.) (39). The results were considered statistically significant whenever the $p$ value was $<0.05$.

\section{RESULTS}

Physiologic variables. Carbon dioxide tension and mean arterial blood pressure were within normal physiologic ranges (34) and did not change during the study periods within or between groups (Table 1). Arterial $\mathrm{pH}$ in the control group was higher at baseline than during reperfusion. Arterial $\mathrm{pH}$ was also higher in the I/R-72 group than in the control group at $15 \mathrm{~min}$ and $2 \mathrm{~h}$ of reperfusion. Arterial $\mathrm{PaO}_{2}$ was higher in the I/R-72 than in the I/R-48 group at baseline, 1 , and $2 \mathrm{~h}$ of reperfusion. Base excess decreased from baseline in the control group during reperfusion, and at 0.25 and $2 \mathrm{~h}$ of reperfusion in the I/R-48 group. Heart rate was lower at 2 and $24 \mathrm{~h}$ of reperfusion than at $0.25 \mathrm{~h}$ of reperfusion within the I/R-48 group and at baseline, 2, 24, 48, and $72 \mathrm{~h}$ than at $0.25 \mathrm{~h}$ of reperfusion within the I/R-72 group. The $\mathrm{pH}, \mathrm{PaO}_{2}$, base excess, and heart rate values were within normal physiologic range, and the small differences among the groups and study periods within the groups were probably not of major physiologic consequences.

Plasma glucose concentrations changed significantly (ANOVA: time within each group, $p<0.05$ ) within $\mathrm{I} / \mathrm{R}-48$ and $\mathrm{I} / \mathrm{R}-72$ groups during the study periods, but not in the control group, and also were higher (ANOVA: interactions versus control group over time, $p<0.05$ ) in the $\mathrm{I} / \mathrm{R}-48$ and $\mathrm{I} / \mathrm{R}-72$ groups than the control group during the study periods (Fig. 1). Plasma lactate concentrations changed during the study periods in the $\mathrm{I} / \mathrm{R}-48$ and $\mathrm{I} / \mathrm{R}-72$ groups (ANOVA: time within each group, $p<0.05$ ), but not the control group. However, only the $\mathrm{I} / \mathrm{R}-72$ group demonstrated significantly higher values than in the control group during the study periods. Although the lactate values appeared slightly higher in the I/R-48 than the I/R-72 group, the differences between the groups were not statistically significant.

All fetal sheep in the I/R-48 and I/R-72 groups, but not in the control sham group, exhibited isoelectric EcoG tracings during the $30 \mathrm{~min}$ of carotid artery occlusion.

Pathology. The pathologic scores of the coronally sliced whole brain sections stained with Luxol fast blue/hematoxylin and eosin demonstrated that the I/R-48 and I/R-72 groups exhibited greater damage across the six brain slices when compared with the slices of the control group (Fig. 2). Each individual slice from the I/R-48 and I/R-72 groups also exhibited greater damage when compared with each respective slice from the control group (Fig. 2). Brain slice 2 exhibited more severe damage in the I/R-72 than the I/R-48 group.

The six slices were averaged to obtain the pathologic scores for the cerebral cortex, white matter, and hippocampus. Results from the averaged scores indicated that the damage was more severe in the white matter of the fetuses exposed to $30 \mathrm{~min}$ of ischemia and $72 \mathrm{~h}$ of reperfusion than the in the fetuses

Table 1. Physiological variables for the study groups by study period

\begin{tabular}{|c|c|c|c|c|c|c|c|c|}
\hline & \multirow[b]{2}{*}{ Group } & \multirow[b]{2}{*}{ Baseline } & \multicolumn{6}{|c|}{ Time after Ischemia/Control Sham Treatment (h) } \\
\hline & & & 0.25 & 1 & 2 & 24 & 48 & 72 \\
\hline \multirow[t]{2}{*}{$\mathrm{pH}$} & Control & $7.35 \pm 0.01$ & $7.30 \pm 0.02 *$ & $7.31 \pm 0.02 *$ & $7.30 \pm 0.01 *$ & $7.32 \pm 0.01 *$ & $7.32 \pm 0.02 *$ & ND \\
\hline & $\mathrm{I} / \mathrm{R}-72$ & $7.35 \pm 0.01$ & $7.35 \pm 0.01 \dagger$ & $7.35 \pm 0.01$ & $7.35 \pm 0.01 \dagger$ & $7.35 \pm 0.01$ & $7.34 \pm 0.01$ & $7.34 \pm 0.01$ \\
\hline \multirow[t]{3}{*}{$\mathrm{PaO}_{2}(\mathrm{kPa})$} & Control & $3.33 \pm 0.13$ & $3.20 \pm 0.13$ & $3.33 \pm 0.13$ & $3.33 \pm 0.13$ & $3.20 \pm 0.13$ & $3.06 \pm 0.13$ & ND \\
\hline & $\mathrm{I} / \mathrm{R}-48$ & $3.20 \pm 0.13$ & $3.46 \pm 0.13$ & $3.20 \pm 0.13$ & $3.20 \pm 0.13$ & $3.33 \pm 0.13$ & $3.20 \pm 0.13$ & ND \\
\hline & $\mathrm{I} / \mathrm{R}-72$ & $3.60 \pm 0.13 \ddagger$ & $3.86 \pm 0.13$ & $3.73 \pm 0.13 \ddagger$ & $3.73 \pm 0.13 \ddagger$ & $3.73 \pm 0.40$ & $3.46 \pm 0.27$ & $3.60 \pm 0.13$ \\
\hline \multirow{2}{*}{$\mathrm{PaCO}_{2}(\mathrm{kPa})$} & $\mathrm{I} / \mathrm{R}-48$ & $7.06 \pm 0.13$ & $6.66 \pm 0.27$ & $6.80 \pm 0.13$ & $6.53 \pm 0.13$ & $6.66 \pm 0.13$ & $6.80 \pm 0.13$ & ND \\
\hline & $\mathrm{I} / \mathrm{R}-72$ & $6.80 \pm 0.13$ & $6.66 \pm 0.13$ & $6.80 \pm 0.13$ & $6.80 \pm 0.13$ & $6.66 \pm 0.27$ & $6.66 \pm 0.27$ & $6.66 \pm 0.13$ \\
\hline \multirow[t]{3}{*}{ Base excess (meq/L) } & Control & $2.4 \pm 1$ & $-1.4 \pm 1^{*}$ & $-1.1 \pm 1^{*}$ & $-1.7 \pm 2 *$ & $0.2 \pm 2^{*}$ & $-1.1 \pm 1^{*}$ & ND \\
\hline & $\mathrm{I} / \mathrm{R}-48$ & $2.3 \pm 1$ & $-0.7 \pm 1 *$ & $0.4 \pm 1$ & $0.5 \pm 1^{*}$ & $1.0 \pm 1$ & $1.0 \pm 1$ & ND \\
\hline & $\mathrm{I} / \mathrm{R}-72$ & $2.5 \pm 1$ & $1.6 \pm 1 \dagger$ & $2.2 \pm 1 \dagger$ & $2.3 \pm 1 \dagger$ & $2.2 \pm 1$ & $1.4 \pm 1$ & $1.6 \pm 1$ \\
\hline \multirow[t]{2}{*}{ Heart rate (beats/min) } & Control & $185 \pm 12$ & $194 \pm 28$ & $167 \pm 9$ & $179 \pm 22$ & $182 \pm 11$ & $192 \pm 24$ & ND \\
\hline & $\mathrm{I} / \mathrm{R}-48$ & $179 \pm 9$ & $205 \pm 13$ & $175 \pm 9$ & $169 \pm 9 \S$ & $180 \pm 10 \S$ & $177 \pm 8$ & ND \\
\hline
\end{tabular}



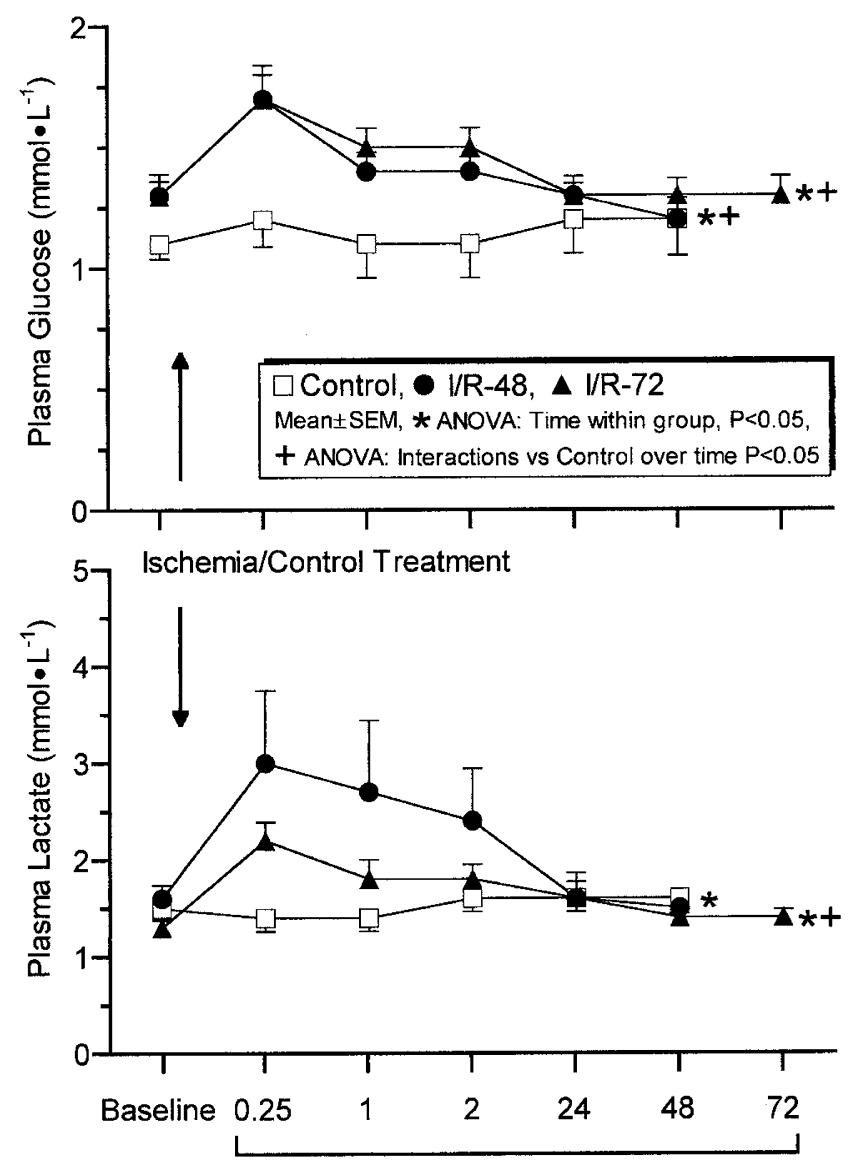

Time after Ischemia/Control Sham Treatment (hrs)

Figure 1. Plasma glucose and lactate concentrations during the study periods in ovine fetuses subjected to control sham treatment (Control, $n=4)$, or 30 min of bilateral carotid artery occlusion and either $48(I / R-48, n=8)$ or $72 \mathrm{~h}$ of reperfusion $(I / R-72, n=10)$. Values are mean \pm SEM. *ANOVA, over time within each group, $p<0.05$; + ANOVA, over time $v s$ control group, $p<$ 0.05 .

exposed control sham treatment (Fig. 3). Injury in the cerebral cortex was significantly greater in the fetuses exposed to 30 min of ischemia and $48 \mathrm{~h}$ of reperfusion than in the control fetuses. Although the injury in the hippocampus appeared greater in the fetuses exposed to $30 \mathrm{~min}$ of ischemia and either 48 or $72 \mathrm{~h}$ of reperfusion, when compared with the control treated fetuses, statistical significance was not achieved, possibly reflecting our small sample size. We cannot rule out the possibility that an increase in the number of animals examined might have clarified whether significant changes might have occurred in the hippocampus and the cerebral cortex at $72 \mathrm{~h}$ of reperfusion.

Characterization of white matter lesions. Representative whole mount coronal brain sections from ovine fetuses subjected to control sham treatment (Control) or to $30 \mathrm{~min}$ of ischemia and either 48 (I/R-48) or 72 (I/R-72) h of reperfusion stained with Luxol fast blue/hematoxylin and eosin are illustrated in Figure 4.

Real color computerized analysis was used to quantify MBP and GFAP within the ischemic lesions. The ischemic lesion was paired with an identical neuroanatomic area from a randomly selected control brain. The ratio of MBP to GFAP
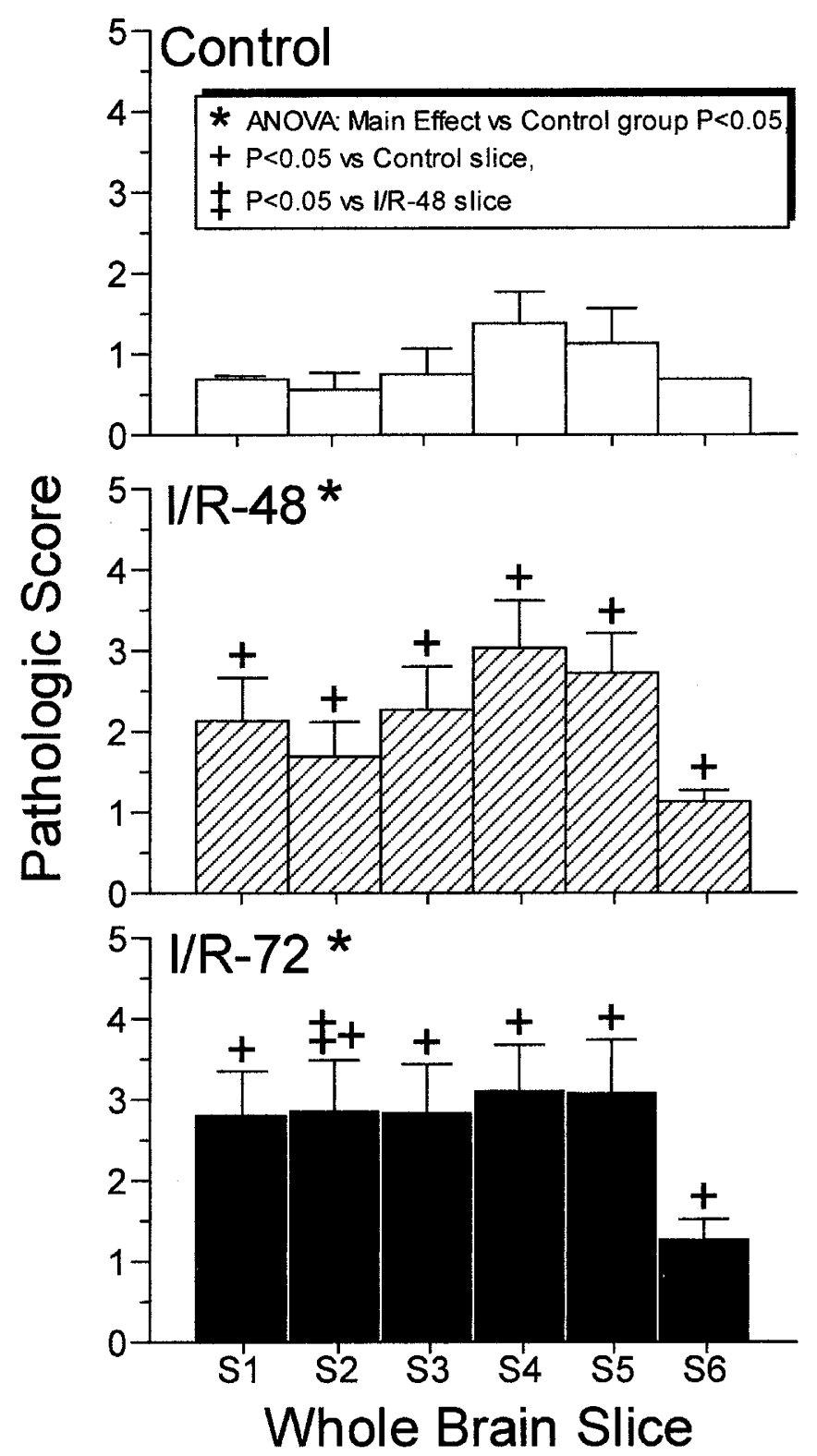

Figure 2. Pathologic scores of the coronally sliced whole brain sections stained with Luxol fast blue/hematoxylin and eosin. All slices received pathologic scores of $0-5$, where $0=0 \%, 1=1 \%$ to $25 \%, 3=51 \%$ to $75 \%, 4=$ $76 \%$ to $95 \%$, and $5=96 \%$ to $100 \%$ of the area damaged. The brain regions represented within each slice are as follows: slice $1(S 1)$ was prefrontal cortex, slice $2(S 2)$ was frontal cortex, slice $3(S 3)$ was posterior medial eminence, slice 4 (S4) was parietal-occipital junction, slice $5(S 5)$ was occipital pole, and slice 6 (S6) was cerebellum and brainstem for the control $(n=4)$, I/R-48 $(n=$ $8)$, and the I/R-72 $(n=10)$ groups. Values are mean \pm SEM. *ANOVA, main effects across brain slices vs control group, $p<0.05 ;+p<0.05$ vs the equivalent slice in the control group; $₫ p<0.05 v$ s the equivalent slice in the I/R-48 group.

decreased as a function of ischemia indicative of a proportionally greater loss of MBP than GFAP (Fig. 5).

$F G F-2$ concentration in frontal cortex. The concentration of FGF-2 was significantly higher in the frontal cortices of the fetuses exposed to $30 \mathrm{~min}$ of ischemia and $72 \mathrm{~h}$ of reperfusion than those of the control fetuses (Fig. 6). The plot of pathologic scores (PS) versus FGF-2 concentrations best fit an expo- 


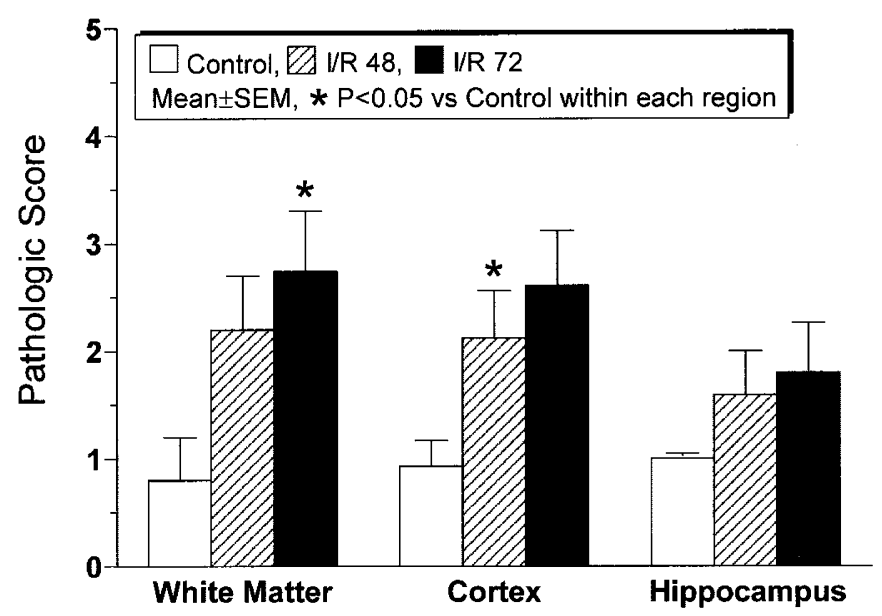

Figure 3. Pathologic scores for white matter, cerebral cortex, and hippocampal damage in the control $(n=4), \mathrm{I} / \mathrm{R}-48(n=8)$, and I/R-72 $(n=10)$ groups. All slices received histologic scores of $0-5$, where $0=0 \%, 1=1 \%$ to $25 \%$, $3=51 \%$ to $75 \%, 4=76 \%$ to $95 \%$, and $5=96 \%$ to $100 \%$ of the area damaged. Values are mean \pm SEM. $* p<0.05$ vs control group within each region.

nential curve $\left(\mathrm{FGF}-2=\mathrm{e}^{(1.6 \mathrm{PS}-0.90)}+743, n=17, r=\right.$ $0.73, p<0.001)$.

\section{DISCUSSION}

We have adapted the method of Gluckman and colleagues (19) to induce cerebral ischemia in fetal sheep by ligation of the vertebral-occipital anastomoses and bilateral occlusion of the carotid arteries. We found evidence of white matter and neuronal injury in fetuses exposed to $30 \mathrm{~min}$ of ischemia and either 48 or $72 \mathrm{~h}$ of reperfusion. Further characterization of the lesions located in the deep white matter revealed a loss of MBP in the ovine fetuses exposed to ischemia and reperfusion. We also found that FGF-2, a purported mediator of recovery from brain injury in adult subjects (22-25), increases in concentration in proportion to the severity of brain damage in the fetus.

In our study, the control sham group exhibited a small amount of damage in the brain regions, particularly in the hippocampus. The brains were removed from the fetus within 8 min after killing of the ewes and placed in formalin after a portion of a frontal lobe had been removed for tissue analysis. Nonetheless, neuronal ischemia is routinely observed in postmortem tissue and represents an unavoidable artifact. Our findings in the control sham group (Figs. 2 and 3) probably reflect this artifact. For this reason, we have included a control group for comparison with the experimental groups. The hippocampus is particularly sensitive to postmortem changes. We cannot rule out the possibility that the lack of significant difference between the control and the I/R-48 and I/R-72 groups in the hippocampus is not related to the baseline damage observed in the control group. Moreover, it remains possible that, if we had been able to examine a larger number of animals, significant changes might have been observed in the hippocampus and/or the cerebral cortex at $72 \mathrm{~h}$ of reperfusion.

Hypoxic/ischemic brain injury during the perinatal period often results in cerebral palsy (40), which may have as its origin in utero ischemic episodes resulting from antepartum or intrapartum events $(41,42)$. The pathologic lesions of cerebral palsy in the developing brain are diverse and include various lesions such as multifocal ischemic injury and periventricular leukomalacia (PVL) (43). Our lesion in the ovine fetus is intragyral and involves the deep and subcortical white matter and the overlying cerebral cortex. Although similar, the white matter lesion that we have identified does not reproduce the classical lesion of PVL, in which the white matter necrosis is confined to the area dorsal and lateral to the external angles of the lateral ventricles (44). However, it does serve as a valid model of focal white matter and cerebral cortical ischemia, which is also found in cerebral palsy.

Several studies have identified white matter injury in the ovine fetus $(10-12,14-16)$. The white matter lesions arising from systemic hypotension were shown to closely resemble the lesion of PVL, because they were localized within the deep white matter around the frontal horns of the foramen of Monro of the lateral ventricles without corresponding neuronal injury (10). Intermittent partial cord occlusion in late-term fetuses for 1 min every 3 min over $2 \mathrm{~h}$ (14) was associated with damage in the intragyral white matter located immediately beneath the cortex. Partial cord occlusion for 60 continuous minutes in fetal sheep at $88 \%$ gestation (15) was associated with considerable variation in injury with damage to the cortex and white matter, ranging from total infarctions of the cerebral cortical and subcortical structures to slight damage to the white matter confined to the junction of the cerebral cortex and the white matter. Increased numbers of GFAP-positive astrocytes and loss of MBP-positive myelin sheaths were also identified, which became accentuated as the injury worsened (15). Lateterm fetal sheep subjected to complete intermittent umbilical cord occlusion (16) had varying amounts of neuronal damage coupled with periventricular white matter lesions. Consistent with these findings, after carotid artery occlusion and $72 \mathrm{~h}$ of reperfusion, we identified and quantified white matter lesions that were intragyral, involved the deep and subcortical white matter and the overlying cerebral cortex, and were associated with relative reductions in MBP within the lesions.

Reddy et al. (12) subjected ovine fetuses at $65 \%$ and $90 \%$ gestation to $30 \mathrm{~min}$ of bilateral carotid occlusion followed by $72 \mathrm{~h}$ of reperfusion and observed damage predominantly in the parasagittal cortex at both ages. In contrast, the preterm fetuses at $65 \%$ of gestation demonstrated rapidly evolving injury resulting in necrosis of the subcortical white matter (12) Although neuronal injury was extensive at both times in gestation, there was only a $20 \%$ occurrence of the subcorticalcortical cystic lesions located between the lateral ventricle and the parasagittal gyrus in the fetuses at $90 \%$ of gestation. In contrast to these findings, we found severe white matter injury in our fetuses at $84 \%$ of gestation that had been exposed to the same insult. The differences between our findings and theirs could, in part, be attributable to differences in the pathologic methods used for analysis of the brain sections. In our study, we found it necessary to stain the coronal brain sections with Luxol fast blue in combination with hematoxylin and eosin to adequately visualize the myelinated areas of the fetal sheep brain. We further characterized the white matter lesions by 

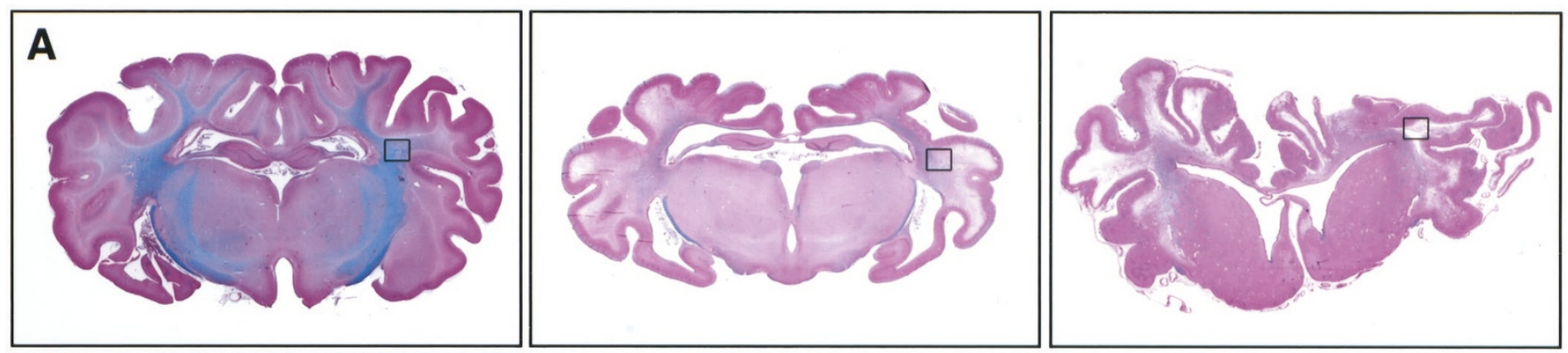

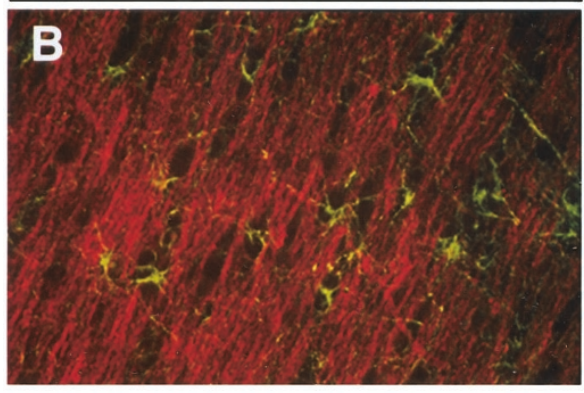

Control

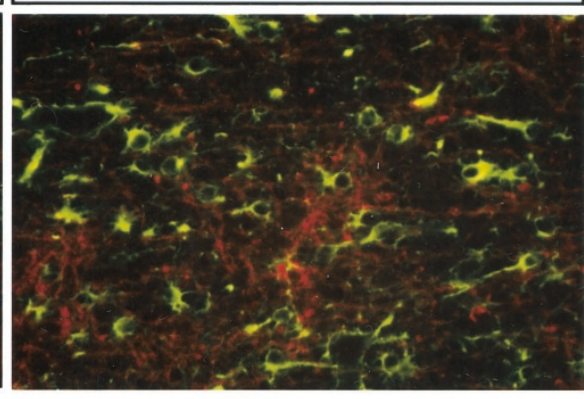

I/R-48

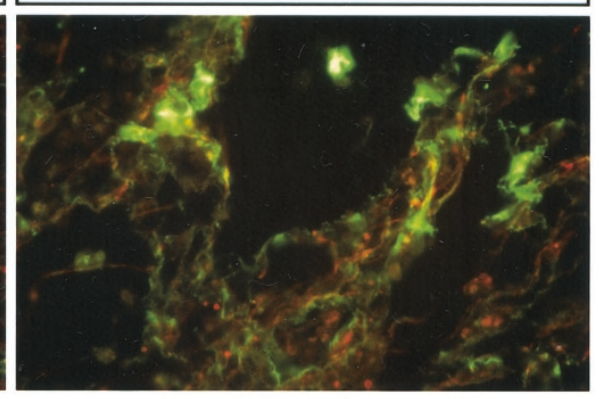

I/R-72

Figure 4. Representative whole mount coronal sections $(A$, top panel, $1 \times)$ from ovine fetuses subjected to control sham treatment $(C o n t r o l)$ or to 30 min of ischemia and either $48(I / R-48)$ or $72(I / R-72)$ hours of reperfusion stained with Luxol fast blue/hematoxylin and eosin. The coronal section from the control group exhibits homogenous blue-stained myelin and healthy-appearing cerebral cortex. In contrast, the sections from the I/R-48 and I/R-72 groups exhibit decreased blue staining, indicating white matter loss, and thinning of the cerebral cortex, indicating neuronal loss. The areas designated within the squares illustrate the regions of the deep white matter from which photomicrographs (B, bottom panel, 250×) were obtained for quantification of glial fibrillary acidic protein (GFAP: green) and myelin basic protein ( $M B P:$ red) using double-indirect immunofluorescence. The next $8-\mu \mathrm{m}$ section immediately adjacent to the section that had been stained with Luxol fast blue/hematoxylin and eosin ( $A$, top panel) was used for the double-indirect immunofluorescence staining and analysis $(B$, bottom panel). In the fetus from the control group, there is an abundance of myelin and the astrocytes appear normal (B, bottom panel, Control). The fetal sheep subjected to ischemia and reperfusion for $48 \mathrm{~h}(B, \mathrm{I} / \mathrm{R}-48)$ and $72 \mathrm{~h}(B, \mathrm{I} / \mathrm{R}-72)$ demonstrated a dramatic reduction in immunocytochemically detectable MBP. Fetal sheep subjected to ischemia and reperfusion for $48 \mathrm{~h}(B, \mathrm{I} / \mathrm{R}-48)$ demonstrated altered cellularity of immunocytochemically detectable GFAP-positive astrocytes, which appeared enlarged and more densely stained. Fetal sheep subjected to ischemia and reperfusion for $72 \mathrm{~h}(B, \mathrm{I} / \mathrm{R}-72)$ demonstrated a paucity of GFAP-positive astrocytes, which appeared abnormally shaped.

double-label fluorescent immunohistochemistry with antibodies to MBP and GFAP. In the study by Reddy et al. (12), a myelin-specific stain was not used, but the cystic infarctions were associated with a macrophage/microglial reaction. Although the methodology of Reddy et al. (12) might have

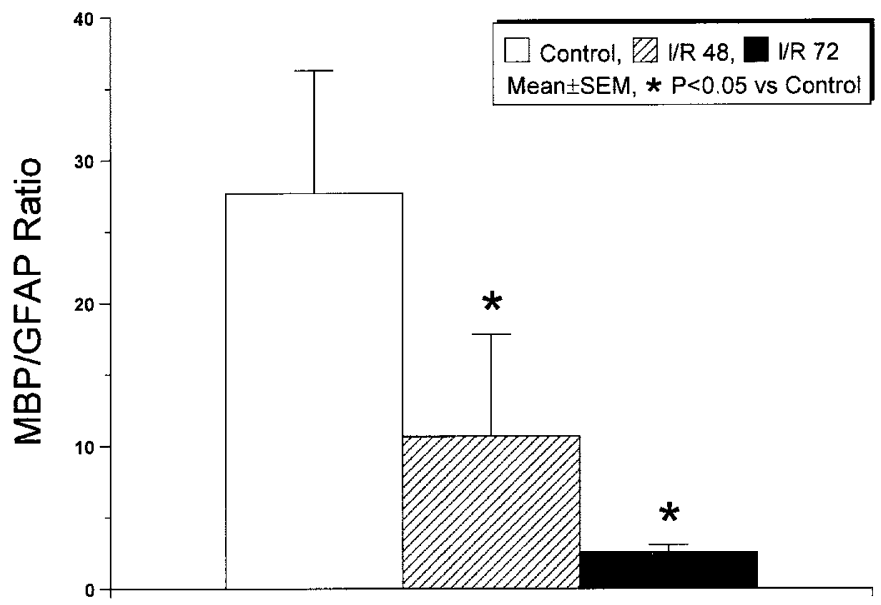

Figure 5. Real color computerized analysis of the ratio of MBP to GFAP immunocytochemically detected in ovine fetal brain. Values are expressed as the ratio of MBP to GFAP within the lesions in the I/R-48 or I/R-72 group or in a neuroanatomically equivalent matched region from a brain of fetus in the control sham group. Values are mean \pm SEM. ${ }^{*} p<0.05 v s$ control group. detected the more severe lesions, it remains possible that the more subtle but consistent lesions that we identified in the deep white matter might have been missed by their analysis. In support of this contention, in a recent study from the same laboratory, when immunohistochemical methods were used to detect the presence and appearance of MBP, a loss of MBP from the white matter was identified in ovine fetuses at $85 \%$

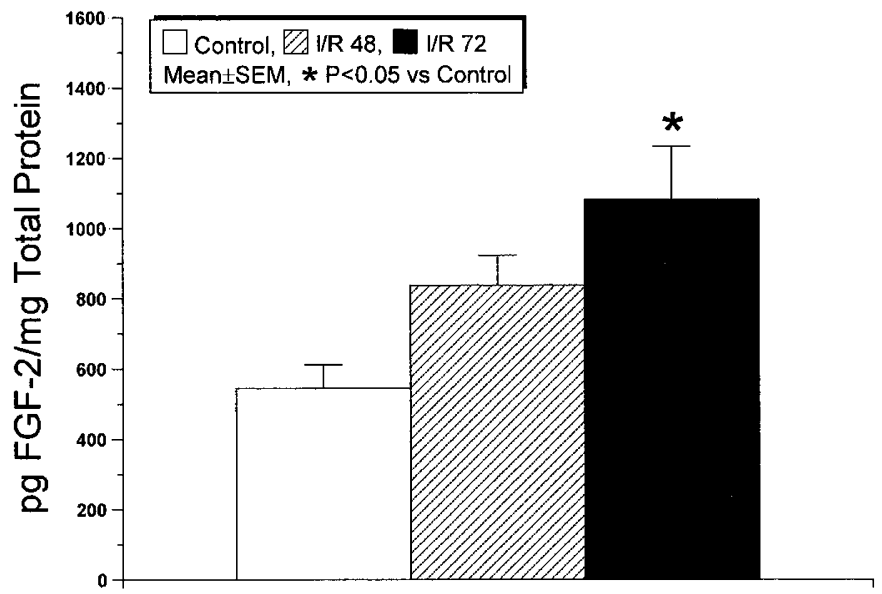

Figure 6. Concentration of FGF-2 (pg FGF-2/mg total protein) in cerebral cortices of ovine fetuses in the control $(n=4)$, I/R-48 $(n=7)$, or the I/R-72 $(n=6)$ groups. Values are mean \pm SEM. ${ }^{*} p<0.05$ vs the control group. 
gestation that had been exposed to $30 \mathrm{~min}$ of bilateral carotid occlusion and $96 \mathrm{~h}$ of reperfusion (11).

During the myelination process, oligodendrocyte differentiation and proliferation precede myelin synthesis and subsequent deposition (45). During oligodendrocyte differentiation and proliferation, the preoligodendrocyte, identified by reactivity to $\mathrm{O} 4 \mathrm{MAb}$, gives rise to the immature oligodendrocyte, which reacts positively to the $\mathrm{O} 1 / \mathrm{O} 4 \mathrm{MAb}(45,46)$. The immature oligodendrocyte, in turn, gives rise to mature oligodendroglia. During myelin synthesis, the mature oligodendrocyte will express MBP and proteolipid protein (PLP) (45). It has recently been proposed that MBP isoforms that contain a key exon-2 sequence, whose expression peaks at times of myelination and remyelination, may play a role in the regulation of myelinogenesis (21). It has been suggested that a key factor in the preponderance of PVL in the premature infant versus a term infant might be due to the vulnerability of actively differentiating oligodendrocytes (47). Consistent with these concepts, when 1-wk-old rat pups were exposed to an acute hypoxic-ischemic insult, degenerative changes were observed in the preoligodendrocyte detected by antibody to $\mathrm{O} 4$ (6). In a recent study (11), a positive correlation was found between the number of PLP mRNA-positive cells and MBP staining in late-gestation fetal sheep subjected to $30 \mathrm{~min}$ of bilateral carotid artery occlusion and $96 \mathrm{~h}$ of reperfusion. Similar to these findings, we found a dramatic decrease in immunocytochemically detectable MBP along with lesions within the Luxol fast blue-stained deep white matter. Luxol fast blue stains phospholipids in the myelin sheath. MBP is a marker for myelin and will only stain processes. The loss of MBP might reflect the loss of processes secondary to target deprivation from neuronal losses. However, the question that remains is what is the effect upon oligodendrocytes per se, and thus the direct effect on white matter cell populations. It would appear that further studies are warranted to examine the expression of O4, O1, PLP, and MBP in the developing ovine brain to correlate the expression of these developmental markers with the timing of maximal vulnerability to ischemic insults.

Several mechanisms may be responsible for the changes that we observed in fetal white matter after ischemia/reperfusion. First, it has been proposed that a state of rapid differentiation in immature oligodendrocytes combined with active myelination might render the developing white matter of the premature fetus inherently vulnerable to ischemia (47). Previous studies have demonstrated that lesions arising from a hypoxicischemic episode are more likely to develop at sites undergoing active myelination (48). Oligodendrocytes have been demonstrated to be vulnerable to the increased concentrations of extracellular glutamate arising from axonal disruption. The transport of glutamate into the cell, the subsequent glutathione depletion, and increased susceptibility to free radical attack are implicated in the oligodendroglial cell death observed after an hypoxic-ischemic insult (49). Mandai et al. (50) postulated that an apoptotic-like process is involved in the cell death of the mature oligodendrocytes. Microglial activation and loss of tropic support (51) as well as cytokine toxicity (52) also have been implicated in the demyelination occurring after an isch- emic insult. Although above mechanisms have been proposed in white matter injury, additional investigations are required to elucidate the pathogenesis of white matter injury in the fetus.

FGF-2 is a purported mediator of recovery from brain injury in adult subjects (22-25). We have examined the effects of in utero ischemia on changes in FGF-2 concentration in the cerebral cortex of fetal sheep and found that the concentration of this growth factor increased in proportion to the severity of brain damage in the fetus. Therefore, we speculate that this factor might have been recruited in an attempt to attenuate the further development of injury in the fetus. FGF-2 has been shown to differentially regulate the development of oligodendrocyte progenitors (53). The increase in FGF-2 that we observed might also be important in rescuing fetal white matter from ischemic damage. However, in the present study, we only examined FGF-2 concentrations in the cerebral cortex.

It is important to point out that the of responses of the fetal brain to in utero ischemia varies with regard to the rank order of increases in the pathologic scores, decreases in the MBP/ GFAP ratio, and increases in the concentration of FGF-2 after ischemia and either 48 or $72 \mathrm{~h}$ of reperfusion. For example, the pathologic scores in white matter are significantly higher than the control values after $72 \mathrm{~h}$ and in the cortex after $48 \mathrm{~h}$ of reperfusion, whereas the ratio of MBP to GFAP is significantly lower than the control values after both 48 and $72 \mathrm{~h}$ of reperfusion. On the other hand, the FGF-2 concentration increases in proportion to the severity of injury and is greatest after $72 \mathrm{~h}$ of reperfusion. The disparity in these responses to ischemia and reperfusion at 48 and $72 \mathrm{~h}$ of reperfusion might have differed if we had been able to study a larger group of fetal sheep, particularly with regard to the pathologic scores in the cortex at $72 \mathrm{~h}$ of reperfusion, which appeared slightly higher at 72 than $48 \mathrm{~h}$ of reperfusion. Alternatively, the pathologic scores in white matter and cortex, the MBP/GFAP ratio, and the concentrations of FGF-2 might truly demonstrate variations in response to the same in utero insult.

In summary, we have identified reproducible white matter and cerebral cortical lesions in ovine fetuses at $84 \%$ of gestation exposed to carotid artery occlusion and reperfusion. The resulting white matter lesions were characterized by a loss of MBP and reactive gliosis. The cortical lesions demonstrated a positive correlation with the increased expression of FGF-2, a purported mediator of recovery from ischemic injury.

Acknowledgments. The authors thank Peter D. Gluckman, D.Sc., and Alister J. Gunn, M.D., for their gracious and invaluable help in setting up their sheep model in our laboratory in the initial phase of these studies. We also thank Christopher Reilly, Timothy Lee, Amy Yuan, and Cathy Yen for their dedication to this project, and the many students who participated in these studies.

\section{REFERENCES}

1. Volpe JJ 1976 Perinatal hypoxic-ischemic brain injury. Pediatr Clin North Am 23:383-397

2. Vannucci RC 1990 Current and potentially new management strategies for perinatal hypoxic-ischemic encephalopathy. Pediatrics 85:961-968

3. Vannucci RC 1993 Mechanisms of perinatal hypoxic-ischemic brain damage. Semin Perinatol 17:330-337 
4. Marcoux FW, Morawetz RB, Crowell RM, DeGirolami U, Halsey JH 1982 Differential regional vulnerability in transient focal cerebral ischemia. Stroke 13:339-346

5. Sheldon RA, Chuai J, Ferriero DM 1996 A rat model for hypoxic-ischemic brain damage in very premature infants. Biol Neonate 69:327-341

6. Jelinski SE, Yager JY, Juurlink BH 1999 Preferential injury of oligodendroblasts by a short hypoxic-ischemic insult. Brain Res 815:150-153

7. Uehara H, Yoshioka H, Kawase S, Nagai H, Ohmae T, Hasegawa K, Sawada T 1999 A new model of white matter injury in neonatal rats with bilateral carotid artery occlusion. Brain Res 837:213-220

8. Pantoni L, Garcia JH, Gutierrez JA 1996 Cerebral white matter is highly vulnerable to ischemia. Stroke 27:1641-1646

9. Penning DH, Grafe MR, Hammond R, Matsuda Y, Patrick J, Richardson B 1994 Neuropathology of the near-term and midgestation ovine fetal brain after sustained in utero hypoxemia. Am J Obstet Gynecol 170:1425-1432

10. Matsuda T, Okuyama K, Cho K, Hoshi N, Matsumoto Y, Kobayashi Y, Fujimoto S 1999 Induction of antenatal periventricular leukomalacia by hemorrhagic hypotension in the chronically instrumented fetal sheep. Am J Obstet Gynecol 181:725-730

11. Guan J, Bennet L, George S, Wu D, Waldvogel HJ, Gluckman PD, Faull RL, Crosier PS, Gunn AJ 2001 Insulin-like growth factor-1 reduces postischemic white matter injury in fetal sheep. J Cereb Blood Flow Metab 21:493-502

12. Reddy K, Mallard C, Guan J, Marks K, Bennet L, Gunning M, Gunn A, Gluckman $\mathrm{P}$, Williams C 1998 Maturational change in the cortical response to hypoperfusion injury in the fetal sheep. Pediatr Res 43:674-682

13. Marumo G, Kozuma S, Ohyu J, Hamai Y, Machida Y, Kobayashi K, Ryo E, Unno N, Fujii T, Baba K, Okai T, Takashima S, Taketani Y 2001 Generation of periventricular leukomalacia by repeated umbilical cord occlusion in near-term fetal sheep and its possible pathogenetical mechanisms. Biol Neonate 79:39-45

14. Clapp JF, Peress NS, Wesley M, Mann LI 1988 Brain damage after intermittent partial cord occlusion in the chronically instrumented fetal lamb. Am J Obstet Gynecol 159:504-509

15. Ikeda T, Murata Y, Quilligan EJ, Choi BH, Parer JT, Doi S, Park SD 1998 Physiologic and histologic changes in near-term fetal lambs exposed to asphyxia by partial umbilical cord occlusion. Am J Obstet Gynecol 178:24-32

16. Ohyu J, Marumo G, Ozawa H, Takashima S, Nakajima K, Kohsaka S, Hamai Y, Machida Y, Kobayashi K, Ryo E, Baba K, Kozuma S, Okai T, Taketani Y 1999 Early axonal and glial pathology in fetal sheep brains with leukomalacia induced by repeated umbilical cord occlusion. Brain Dev 21:248-252

17. Mallard EC, Williams CE, Gunn AJ, Gunning MI, Gluckman PD 1993 Frequent episodes of brief ischemia sensitize the fetal sheep brain to neuronal loss and induce striatal injury. Pediatr Res 33:61-65

18. Williams CE, Gunn AJ, Synek B, Gluckman PD 1990 Delayed seizures occurring with hypoxic-ischemic encephalopathy in the fetal sheep. Pediatr Res 27:561-565

19. Williams CE, Gunn AJ, Mallard C, Gluckman PD 1992 Outcome after ischemia in the developing sheep brain: an electroencephalographic and histological study. Ann Neurol 31:14-21

20. Petito CK, Morgello S, Felix JC, Lesser ML 1990 The two patterns of reactive astrocytosis in postischemic rat brain. J Cereb Blood Flow Metab 10:850-859

21. Pedraza L, Fidler L, Staugaitis SM, Colman DR 1997 The active transport of myelin basic protein into the nucleus suggests a regulatory role in myelination. Neuron 18:579-589

22. Kiyota Y, Takami K, Iwane M, Shino A, Miyamoto M, Tsukuda R, Nagaoka A 1991 Increase in basic fibroblast growth factor-like immunoreactivity in rat brain after forebrain ischemia. Brain Res 545:322-328

23. Takami K, Kiyota Y, Iwane M, Miyamoto M, Tsukuda R, Igarashi K, Shino A, Wanaka A, Shiosaka S, Tohyama M 1993 Upregulation of fibroblast growth factorreceptor messenger RNA expression in rat brain following transient forebrain ischemia. Exp Brain Res 97:185-194

24. Speliotes EK, Caday CG, Do T, Weise J, Kowall NW, Finklestein SP 1996 Increased expression of basic fibroblast growth factor (bFGF) following focal cerebral infarction in the rat. Brain Res Mol Brain Res 39:31-42

25. Iwata A, Masago A, Yamada K 1997 Expression of basic fibroblast growth factor mRNA after transient focal ischemia: comparison with expression of c-fos, c-jun, and hsp 70 mRNA. J Neurotrauma 14:201-210

26. Nozaki K, Finklestein SP, Beal MF 1993 Basic fibroblast growth factor protects against hypoxia-ischemia and NMDA neurotoxicity in neonatal rats. J Cereb Blood Flow Metab 13:221-228
27. Kirschner PB, Henshaw R, Weise J, Trubetskoy V, Finklestein S, Schulz JB, Beal MF 1995 Basic fibroblast growth factor protects against excitotoxicity and chemical hypoxia in both neonatal and adult rats. J Cereb Blood Flow Metab 15:619-623

28. Barth A, Barth L, Morrison RS, Newell DW 1996 bFGF enhances the protective effects of MK-801 against ischemic neuronal injury in vitro. Neuroreport 7:14611464

29. Jiang N, Finklestein SP, Do T, Caday CG, Charette M, Chopp M 1996 Delayed intravenous administration of basic fibroblast growth factor (bFGF) reduces infarct volume in a model of focal cerebral ischemia/reperfusion in the rat. J Neurol Sci 139:173-179

30. Ren JM, Finklestein SP 1997 Time window of infarct reduction by intravenous basic fibroblast growth factor in focal cerebral ischemia. Eur J Pharm 327:11-16

31. Bethel A, Kirsch JR, Koehler RC, Finklestein SP, Traystman RJ 1997 Intravenous basic fibroblast growth factor decreases brain injury resulting from focal ischemia in cats. Stroke 28:609-615

32. Mallard EC, Williams CE, Johnston BM, Gluckman PD 1995 Neuronal damage in the developing brain following intrauterine asphyxia. Reprod Fertil Dev 7:647-653

33. Jackson BT, Egdahl RH 1960 The performance of complex fetal operations in utero without amniotic fluid loss or other disturbances of fetal-maternal relationships. Surgery 48:564-570

34. Stonestreet BS, Le E, Berard DJ 1993 Circulatory and metabolic effects of betaadrenergic blockade in the hyperinsulinemic ovine fetus. Am J Physiol 265:H1098H1106

35. Gunn AJ, Gunn TR, de Haan HH, Williams CE, Gluckman PD 1997 Dramatic neuronal rescue with prolonged selective head cooling after ischemia in fetal lambs. J Clin Invest 99:248-256

36. Brown AW, Brierley JB 1972 Anoxic-ischaemic cell change in rat brain light microscopic and fine-structural observations. J Neurol Sci 16:59-84

37. Faris RA, McBride A, Yang L, Affigne S, Walker C, Cha CJ 1994 Isolation, propagation, and characterization of rat liver serosal mesothelial cells. Am J Pathol 145:1432-1443

38. Ott RL, Longnecker M 2001 Statistical Methods and Data Analysis. Duxbury, Pacific Grove, CA, pp 1025-1045

39. Institute SASP 1990 SAS/STAT User's Guide. SAS Institute, Cary, NC, pp 891-997

40. Vannucci RC, Perlman JM 1997 Interventions for perinatal hypoxic-ischemic encephalopathy. Pediatrics 100:1004-1014

41. Hill A, Volpe JJ 1981 Seizures, hypoxic-ischemic brain injury, and intraventricular hemorrhage in the newborn. Ann Neurol 10:109-121

42. Shields JR, Schifrin BS 1988 Perinatal antecedents of cerebral palsy. Obstet Gynecol 71:899-905

43. Volpe J 1995 Hypoxic-ischemic encephalopathy. In: Neurology of the Newborn. WB Saunders, Philadelphia, PA, pp 260-313

44. Banker BQ, Larroche JC 1962 Periventricular leukomalacia of infancy. Arch Neurol $7: 386-410$

45. Kinney HC, Back SA 1998 Human oligodendroglial development: relationship to periventricular leukomalacia. Semin Pediatr Neurol 5:180-189

46. Back SA, Luo NL, Borenstein NS, Levine JM, Volpe JJ, Kinney HC 2001 Late oligodendrocyte progenitors coincide with the developmental window of vulnerability for human perinatal white matter injury. J Neurosci 21:1302-1312

47. Volpe JJ 1992 Brain injury in the premature infant-current concepts of pathogenesis and prevention. Biol Neonate 62:231-242

48. Rice III JE, Vannucci RC, Brierley JB 1981 The influence of immaturity on hypoxic-ischemic brain damage in the rat. Ann Neurol 9:131-141

49. Oka A, Belliveau MJ, Rosenberg PA, Volpe JJ 1993 Vulnerability of oligodendroglia to glutamate: pharmacology, mechanisms, and prevention. J Neurosci 13:1441-1453

50. Mandai K, Matsumoto M, Kitagawa K, Matsushita K, Ohtsuki T, Mabuchi T, Colman DR, Kamada T, Yanagihara T 1997 Ischemic damage and subsequent proliferation of oligodendrocytes in focal cerebral ischemia. Neuroscience 77:849-861

51. Shuman SL, Bresnahan JC, Beattie MS 1997 Apoptosis of microglia and oligodendrocytes after spinal cord contusion in rats. J Neurosci Res 50:798-808

52. Ye P, D'Ercole AJ 1999 Insulin-like growth factor I protects oligodendrocytes from tumor necrosis factor-alpha-induced injury. Endocrinology 140:3063-3072

53. Gard AL, Pfeiffer SE 1993 Glial cell mitogens bFGF and PDGF differentially regulate development of $\mathrm{O}^{+} \mathrm{GalC}^{-}$oligodendrocyte progenitors. Dev Biol 159:618-630 